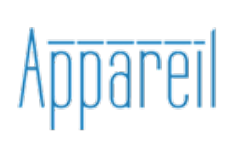

Appareil

$12 \mid 2013$

Walter Benjamin. Politiques de l'image

\title{
La moralité, le droit et la place de la critique : « La signification du temps dans le monde moral » de Walter Benjamin
}

\section{Andrew Benjamin}

Traducteur : Germinal Pinalie

\section{(2) OpenEdition}

\section{Journals}

Édition électronique

URL : http://journals.openedition.org/appareil/1948

DOI : 10.4000/appareil.1948

ISSN : 2101-0714

Éditeur

MSH Paris Nord

Référence électronique

Andrew Benjamin, «La moralité, le droit et la place de la critique : «La signification du temps dans le monde moral » de Walter Benjamin », Appareil [En ligne], 12 | 2013, mis en ligne le 13 décembre 2013, consulté le 30 juillet 2020. URL : http://journals.openedition.org/appareil/1948 ; DOI : https://doi.org/ 10.4000/appareil. 1948

Ce document a été généré automatiquement le 30 juillet 2020.

\section{c) (†) $\odot$}

Appareil est mis à disposition selon les termes de la Licence Creative Commons Attribution - Pas d'Utilisation Commerciale - Pas de Modification 4.0 International 


\title{
La moralité, le droit et la place de la critique : « La signification du temps dans le monde moral » de Walter Benjamin
}

\author{
Andrew Benjamin \\ Traduction : Germinal Pinalie
}

\section{NOTE DE L'AUTEUR}

Je remercie mes collègues Franz-Josef Dieters et Kate Rigby pour avoir bien voulu discuter de certains aspects de ce texte avec moi. Par ailleurs, je voudrais citer l'aide fournie par Anthony Phelan. J'aimerais également remercier mes étudiants qui ont participé à un séminaire sur Walter Benjamin tenu à la Monash University de mars à mai 2010. Le séminaire s'est concentré sur ces textes de Benjamin : The Meaning of Time in the Moral World, Towards a Critique of Violence et Theological-Political Fragment. Le travail issu du séminaire sur ce dernier texte a déjà été publié. Voir mon article : "Messianic Nature: Notes on Walter Benjamin's Theological-Political Fragment”, in Limbus: Australisches Jahrbuch für germanistische Literatur und Kulturwissenschaft, Australian Yearbook of German Literary and Cultural Studies, 2010.

En même temps que la critique joue un rôle fondamental au sein de la philosophie moderne, elle apporte en premier lieu une profonde division entre un souci des conditions d'existence, de là l'héritage kantien, et en second lieu des stratégies philosophiques qui ne peuvent pas être séparées de diverses modalités d'interruption et de commencement. Bien qu'il s'agisse d'une division qui rend possibles des moments de connexion instructifs, c'est le second sens de critique qui sera ici en point de mire. Un exemple clair d'une telle compréhension de la critique, liée à la destruction, se 
trouve au commencement de la première des Méditations métaphysiques de Descartes. Descartes définit sa stratégie dans les termes suivants :

Je me suis procuré un repos assuré dans une paisible solitude, je m'appliquerai sérieusement et avec liberté à détruire généralement toutes mes anciennes opinions ${ }^{2}$.

2 Ainsi, ce qui fait partie intégrante du projet de Descartes est double. Il y a, en premier lieu, cessation, pensée comme destruction, et, en second lieu, un commencement fondé sur cette destruction. La tradition, la philosophie (et sa relation avec la science) et enfin Dieu n'étaient, pour Descartes, pas immunisés contre le processus du doute. De cette façon, Descartes était en mesure de mettre en scène une re-fondation du philosophique sur la base de l'interruption radicale que le doute établit. Si la position de Descartes est essentiellement moderne en ce qu'elle rassemble une fin, comprise comme une forme d'interruption, et un commencement qui assure, ou cherche à assurer la nature effective de cette interruption, alors elle est aussi prémoderne en ce que sa pensée de l'interruption ne suppose pas une mise en scène structurée par un déséquilibre de puissance, ni ne rejette toute compréhension de ce que l'interruption ne peut opérer seulement au niveau du conceptuel. L'interruption nécessite une philosophie de l'histoire (et donc une conception du temps historique) comprenant une double détermination. La première serait une détermination dans laquelle l'interruption ellemême peut être pensée, et alors secondairement, cette détermination amène la reconnaissance concomitante de ce qu'une telle pensée de l'interruption est par sa nature même contestable au niveau fondamental, précisément parce qu'il y a un déséquilibre de puissance. Cette dernière position établit la simplicité inhérente à la formulation de la destruction par Descartes. Ce serait comme si la destruction ne laissait pas de reste, et que la destruction était elle-même un processus non problématique dans lequel les recours à la raison étaient tenus pour persuasifs et, en tant que tels, mettaient sur la touche toute possibilité que les déséquilibres de puissance puissent jouer le rôle de limites.

3 La limite de Descartes ne se trouve pas au sein de l'identification d'une relation entre destruction et commencement. Ce qui importe, c'est comment comprendre la relation. La destruction est un motif important à l'intérieur de différentes conceptions du philosophique. Elle permet, dans un premier temps, d'établir un lien entre Descartes, Heidegger et Walter Benjamin, dans la mesure où tous envisageaient le dépassement de la tradition au nom d'une autre inauguration philosophique. Dans un second temps, elle indique où les points de différenciation doivent être cherchés ${ }^{3}$. Les différences entre leurs conceptions du philosophique trouvent leurs sources dans la façon dont la destruction est comprise et l'inauguration mise en scène, et par là comment le présent, au lieu de la destruction et de l'inauguration, est lui-même interprété. Cependant, la valeur et la limite de Descartes peuvent toutes deux être situées dans l'insistance sur la nécessité de la destruction, à ce titre il ne peut y avoir d'autre commencement que celui fondé sur un mode de destruction. La limite, qui est tout autant instructive, concerne l'interaction entre ce qui pourrait être décrit, comme la relation entre pouvoir et temps. La signification de l'œuvre de Walter Benjamin est qu'elle peut être située à un moment précis, c'est-à-dire à une intersection composée d'abord du lien de la destruction et de l'inauguration d'un côté, de ce qui peut être décrit de la meilleure façon comme une politique du temps d'un autre côtét. Ce moment fournit la structure de la critique. 
4 Afin de parvenir à une meilleure compréhension des façons dont ce moment opère dans les écrits de Walter Benjamin, plutôt que de tenter de fournir une vision synoptique, l'accent sera mis sur un texte spécifique, à savoir The Meaning of Time in the Moral World ${ }^{5}$. Ce texte a été écrit au moment même où Benjamin était en train de concevoir le détail de son texte décisif sur la relation entre le droit et la politique, Towards a Critique of Violence ${ }^{6}$. Le premier texte n'a pas été publié à l'époque. Cependant, il a été écrit en 1921, la même année que Towards a Critique of Violence. La raison pour laquelle on peut centrer le projet de développer le lien entre critique et destruction autour de ce texte est, qu'en dépit de sa brièveté, il met en scène des éléments fondamentaux de la philosophie politique de Benjamin, et ainsi de la conception de la critique que l'on peut voir émerger de ses écrits. Il y a trois éléments centraux pour son projet d'ensemble qui trouvent diverses formes d'expression dans ce court texte. Le premier est la suspension du droit au nom de la moralité; la moralité en tant qu'ouverture à la justice. Le deuxième est la reconnaissance du fait qu'un tel rapport au droit implique la création d'une ouverture dont le contenu ne peut être déterminé à l'avance. En tant que tel, il fait advenir un sens de médiation. La suspension du droit est la suspension de l'immédiateté. La création des dimensions spatiale et temporelle de la médiation est un geste fondamental pour une politique du temps. Le troisième élément est qu'une telle ouverture, et donc ce qu'elle rend possible, a ses conditions de possibilité dans une potentialité déjà présente au sein du monde. De là, il est implicite dans ce dernier élément que le rapport de la destruction à une inauguration fondatrice est présent, et ce nécessairement, comme une potentialité non encore réalisée ${ }^{7}$.

Comme prélude à l'étude détaillée du texte de Benjamin, il est vital qu'une attention encore plus grande soit portée à la façon dont la «destruction " peut être reconçue comme ayant un rapport fondateur avec à la fois la possibilité d'une pensée philosophique de la politique et une conception de la potentialité. Alors que Benjamin ne se réfère pas à Marx à ce propos (même si dans ses écrits postérieurs les références à Marx deviennent de plus en plus détaillées et nuancées), il y a une suggestion importante sur la manière dont une conception de la destruction, comme liée de façon inhérente à la potentialité, pourrait être comprise à travers les écrits de Marx luimême. Ceci se trouve à la fin de la onzième des Thèses sur la philosophie de Feuerbach. Alors que d'un côté le langage du texte est raisonnablement simple dans sa juxtaposition de l'interprétation et du changement, on peut suggérer que le langage de la juxtaposition contient le problème même que sa mise en scène aurait toujours nécessité. Marx écrit ceci :

Die Philosophen haben die Welt nur verschieden interpretiert; es kommt aber darauf an, sie zu verändern 8 .

6 La traduction traditionnelle de cette ligne célèbre, si elle n'est pas incorrecte, est contrainte d'exclure d'autres possibilités contenues dans l'original, en prenant la forme suivante :

Les philosophes n'ont fait qu'interpréter le monde de différentes manières, ce qui importe c'est de le transformer'.

7 Dans la forme manuscrite originale, les mots interpretiert et verändern étaient soulignés. En dépit de la traduction traditionnelle, ce n'est pas comme si ce qui est juxtaposé ici était l'inaction et l'action. Ce que ce cadre ouvre, en tant que question, est la signification de verändern. Une partie de la réponse se trouve dans l'incorporation dans ce terme de anderer («autre»). De plus, une autre partie de la réponse doit être 
entrevue dans le sens implicite de procès que contient le terme (de là le déplacement toujours possible de verändern à Veränderung). Par ailleurs, plutôt que de suggérer que tout ce qui est en jeu dans la Thèse est la possibilité du changement, là où le changement et donc la destruction pourraient être assimilés à des formes variables de modification, comme si le changement pouvait être subsumé sous l'intitulé d'« adaptation" (Anpassung) ou de "transformation " (Verwandlung), une fois que la centralité est attribuée à la présence de anderer, alors la Thèse elle-même peut être comprise comme une juxtaposition de l'«interprétation" au processus qui rend le monde autre. Et il est à noter que ce qui est en jeu est une pensée du «monde» (die Welt), le terme étant présent à la fois chez Marx et Benjamin ("Le monde » (die Welt) est, après tout, nommé dans le titre du texte de Benjamin). Ce qui doit être rendu autre est le monde. Telle que l'interprète la Thèse, «l'interprétation » accepte le fait que le monde est donné. Le mouvement contraire est le fait de rendre le monde autre. De là, la question : que signifie le fait de rendre le monde autre ? Tenter de répondre à cette question nécessite de situer le domaine de réponse.

Pour commencer, il faut reconnaître que le fait de rendre autre est un processus. Cependant, en tant que processus, il a besoin de ce moment durant lequel il est luimême inauguré. L'inauguration n'est possible que si la potentialité d'être autre est déjà là. Cette potentialité serait déjà présente au sein de ce qui peut être décrit comme la qualité de il y a du monde. L'interprétation, en tant qu'elle est utilisée dans sa juxtaposition avec le fait de rendre autre à l'intérieur de la Thèse, présuppose que la qualité de il y a du monde coïncide effectivement avec le monde. L'interprétation est ainsi liée à un genre d'empirisme. À l'opposé de cette position, on peut arguer que la compréhension par Marx du fait de rendre autre présuppose que la présence matérielle du monde, en tant que force immatérielle, apporte sa capacité à être autre. Le fait, pour le monde, d'être rendu autre qui en résulte est la continuité du processus, un processus qui demande sa forme d'inauguration, et donc la mise en scène de la discontinuité, dans laquelle cette potentialité continue d'être réalisée. Le fait de rendre le monde autre prend ainsi la forme d'un événement complexe. Cela demande un sens de l'externalité dans lequel il y a un genre de continuité qui ne peut être fondée sur une logique propre au monde. Ce que cela signifie est qu'une partie de cet événement est la présence d'une contre-strophe, qui serait donc présente en tant que catastrophe productive. La catastrophe doit donc être pensée en termes de coprésence de la continuité et de la discontinuité ${ }^{10}$. Mais, en même temps, la catastrophe comme présence effective demande une conception spécifique de l'internalité. Cette dernière internalité est là en tant que potentialité au sein du monde, qui implique que le monde aura toujours contenu une capacité à être autre (quand bien même, à un moment spécifique, cette capacité n'est pas reconnue, ou a même pu lui être déniée). Le fait de rendre autre, ainsi, ne signale pas la présence d'un événement requérant une forme de pure externalité. Au contraire, le fait de rendre autre nécessite des formes de relation. De plus, le fait de rendre autre nécessite également une conception de la valeur. La valeur, cependant, n'est située ni au sein d'un sujet ni dans ce qui pourrait être extrait d'une subjectivité abstraite. Le lieu de la valeur se trouve dans le monde (quoiqu'en tant que potentialité). Il est ici vital d'ajouter l'additionnel mais néanmoins implicite point qui tient qu'au sein de la modernité, comme une part de son autodéfinition, la présence du monde en tant que lieu de la valeur est inscrite dans la réalité incontournable du fait que le monde (la Welt de Marx) est structuré par la présence effective du déséquilibre des relations de pouvoir. Ainsi donc, la vie mondaine est à la fois la présence effective 
d'un jeu donné de relations de pouvoir, et également ce qui peut être rendu autre. Le fait de rendre autre devient un autre jeu de telles relations. Ce que cela signifierait, dans les termes mêmes de Benjamin, c'est que le «monde du droit » (die Welt des Rechts) céderait sa place au « monde moral » (die moralische Welt).

Ce qui est posé ici est qu'une des façons les plus productives de formuler les enjeux centraux du projet philosophico-politique de Benjamin, tel que ce projet se déploie dans les textes écrits autour de l'époque de la Critique de la violence, est de formuler ce projet en termes de rapport entre externalité et internalité, comme nous venons de le voir. Ce qui est ici au travail est donc une pensée de l'événement incluant, d'un côté, une forme de relation, et, de l'autre, une conception de l'interruption comme étant ce qui fonde. Cette double conception de l'événement fournit la scène dans laquelle localiser la force du texte de Benjamin, et en même temps de plus amples détails sur le moment dans lequel la structure de la critique est située.

1 Le court texte de Benjamin intitule The Meaning of Time in the Moral World s'ouvre sur une considération sur le rapport entre le droit et les institutions légales, et ce qu'il appelle «les temps depuis longtemps révolus ». On pensait, suggère Benjamin, que le droit et les institutions légales avaient un rapport déjà donné à la "moralité ». Et pourtant, affirme-t-il, ce n'est pas le cas. Il continue en arguant que :

Or le pouvoir du droit sur ce lointain passé et l'intérêt qu'il lui porte résulte d'une tendance qui, bien loin de représenter la présence en lui de la morale, trace au contraire très précisément la frontière qui sépare le droit [Recht] du monde moral; la tendance à la vengeance [Vergeltung] ${ }^{11}$.

L'évocation de la "vengeance " convoque le domaine du destin et donc l'oeuvre de l'immédiateté. Dans "Destin et caractère", écrit deux ans avant le fragment sur le monde moral, le « destin » est inextricablement lié à une forme de continuité imposée puis naturalisée. Il y a un élément additionnel qui, dans ce contexte, est décisif. Le destin définit également le domaine de la "culpabilité », un domaine dans lequel la « culpabilité » est imposée à la vie. L'interruption de l'œuvre de la culpabilité arrive dans un contexte, comme exposé dans "Destin et caractère ", dans lequel la confusion erronée de la « justice » (Gerechtigkeit) avec « l'ordre du droit » (die Ordnung des Rechts) a été identifiée - une identification qui permet de séparer la justice du droit - et qui situe la possibilité pour l'interruption du destin sous la forme de l'action. Cette interruption ne s'est pas produite au sein du droit. Elle prend ses distances avec le droit en advenant. En effet, sa situation était bien extérieure au droit (ou tout au moins à cette conception du droit qui peut être différenciée de la justice). À ce sujet, Benjamin écrit :

Ce ne fut pas dans le droit, mais dans la tragédie, que la tête du Génie émergea pour

la première fois de la brume de la faute, car dans la tragédie, le destin démonique se trouve battu en brèche ${ }^{12}$.

Les deux moments signifiants sont ici l'élévation de la tête et la présence d'une percée. Les deux doivent être comprises comme des figures de l'interruption. Un autre nom pour la mise en scène de cette sortie est «chance» (Glück). À ce sujet, Benjamin est explicite: "Le bonheur est bien plutôt ce qui libère l'homme de l'enchaînement des destins et du filet de son propre destin ${ }^{13} »$. Dans les trois exemples, ce qui est mis en scène est une interruption qui doit être comprise comme une ouverture. L'ouverture est productive. Elle donne lieu, et donc rend possible. 

dressée. La « vengeance » en tant qu'acte qui, ayant été décrété à l'avance, est le refus de la médiation, au point que celle-ci est comprise comme la dimension spatiotemporelle de la décision, et donc du jugement. Alors que ce qui est identifié comme « vengeance » est pragmatiquement soumis aux limites contemporaines et temporelles au sein de la pratique légale - par exemple, un statut de la prescription - il n'en reste pas moins que ce que Benjamin nomme le "pouvoir de la vengeance" (vergeltende Gewalt) peut continuer de prévaloir d'une génération à l'autre, tel un déroulement fatidique. Certes, si ce n'est pas l'argument de Benjamin lui-même, il est possible cependant de suggérer qu'une vision moderne de la vengeance apparaît dans la nécessité apparente de lier le droit et la peine. Pour Benjamin pourtant, la vengeance comme force structurante est "indifférente au temps». En outre, le "Jugement dernier » (das jüngste Gericht) est identifié au moment final de la " vengeance », et donc la fin au lieu d'un jugement et d'une peine d'une portée globale et totale (où la position du sujet défini en termes de culpabilité a sa présence la plus insistante). Ce qui importe ici est que, pour Benjamin, le lien entre le Jugement dernier et le moment où la vengeance se voit laisser libre cours, et où ainsi le domaine de l'immédiateté est ouvert, manque pour comprendre ce qu'il décrit comme «l'incommensurable importance du Jugement dernier ». Avant de s'intéresser à la question de cette "importance », il est essentiel de noter la distinction entre immédiateté et médiation car les deux termes et la façon dont ils diffèrent joueront un rôle fondamental dans l'argumentation qui va être développée dans cet article.

L'immédiateté opère dans le rapport entre le destin et le droit; en outre, elle suppose une position de sujet qui est structuré par ce rapport. Elle envisage également, à la fois au regard de ce sujet et de la conception du temps historique dans laquelle est située cette structure, d'apporter, comme cela a déjà été indiqué, une position de sujet défini par un sentiment de culpabilité originelle (par exemple, celui d'avoir été voué). La vengeance, qu'elle soit présente en termes de droit ou de conception spécifique de la justice, se produit au sein de la structure créée par le destin et la culpabilité. Par conséquent, ce qui est à l'œuvre ici doit être compris comme nécessitant la présence d'une réponse déjà (pré)déterminée à un sujet déjà donné et déterminé. Étant données les structures de ce cadre, la justice en viendrait à être mise en équation avec la vengeance, positionnement qui a une réciprocité presque nécessaire, puisqu'une telle équation interdit évidemment la possibilité de la médiation. La médiation est écartée précisément parce que la présence opérative de ce qui est toujours déterminé à l'avance (par exemple, l'œuvre du destin et la structure de la culpabilité) clôt le cadre dans lequel la médiation aurait pu se produire. La médiation, par conséquent, situe le sujet dans un cadre qui n'est pas déterminé en premier par une structure originelle de culpabilité, et secondairement par une conception de la justice dans laquelle la justice est mise en équation avec la vengeance. Le projet d'une « moralité », de la manière dont le terme est utilisé dans le texte de Benjamin, nécessite de libérer la justice d'un tel cadre. En allant plus loin - dans ce qui serait le résultat d'une suspension de ce cadre la justice ne pourrait plus être mise en équation avec un acte immédiat et unique, si cet acte est compris comme ayant une finalité d'auto-accomplissement. Mieux : la justice est ce qui est toujours et par nécessité un souci permanent. La justice porte donc avec elle l'inévitabilité de formes de contestation. En tant que telle, la médiation n'est donc pas seulement le dépassement de l'acte singulier; la médiation exige un sens du placement, de l'espace, dans lequel la justice comme processus peut avoir lieu et être 
mise en pratique (le fait de rendre autre est une présence sur une telle scène). Dans la médiation, la justice nécessite des positions de sujet qui sont définies autant par des points communs que par leur emplacement. L'immédiateté implique l'acte singulier d'un Dieu (ou d'un dictateur) alors que la médiation implique la temporalité, et donc la présence institutionnelle de lieux de délibération (au sein de celle-ci l'ontologie de l'être humain, l'être de l'être humain, en viendrait à être définie par des voies qui rendent l'emplacement central, donc comme être-en-place).

Après avoir remarqué la force de la distinction entre immédiateté et médiation, il devient possible de revenir à la manière dont cette distinction est à l'œuvre, quoique implicitement, dans la tentative de Benjamin de distinguer le «droit » du « monde de la moralité ». En effet, il devient possible d'aller plus loin et de suggérer que la centralité du processus et donc l'œuvre de la médiation est déjà là dans le texte de Benjamin. Elle se trouve dans la ligne qui donne son titre au texte. Benjamin est en fait préoccupé par «le sens du temps dans l'économie du monde moral ». L'évocation de « l'économie » est l'accent mis sur l'inéliminabilité du processus.

Pour en revenir à la question de l'importance du Jugement dernier, il est à noter qu'elle se pose lorsque son identification, pensée en termes de "vengeance » et de droit, est défaite, suspendue, au nom d'une qualité qui est impensable au sein du domaine du droit, à savoir «le pardon» (Vergebung). Ce qui se résume, évidemment, à l'immédiateté cédant sa place à la médiation, à travers ce qui émergera comme la suspension de la première ; suspension en tant que forme de destruction. En effet, telle est l'importance du Jugement dernier, mais seulement lorsqu'il est compris comme le jour qui est "constamment reporté ». Avec ce déplacement de la "vengeance » au "pardon ", et une fois que la nécessité du report ou du renvoi est introduite, ce qui est alors mis en scène est le mouvement du " monde du droit » vers le «monde moral ». La suspension du droit, le moment qui peut être compris comme une forme de destruction, est l'inauguration $d u$ « monde moral ». C'est à ce point du développement de l'argument général du texte de Benjamin qu'émerge la figure d'Atê. Le passage complet du texte de Benjamin est le suivant :

Mais le pardon trouve dans le temps (in der Zeit) la configuration et la puissance qui lui permettent de lutter contre la vengeance. Car Atê poursuit le criminel dans un temps qui est, non le calme plat et solitaire de l'angoisse, mais la bruyante tempête du pardon qui souffle en rafales hurlantes depuis le jugement qui se rapproche sans cesse et contre laquelle Atê ne peut rien ${ }^{14}$.

Voici le moment crucial. Un repositionnement du temps a lieu. Le temps est désormais en place, et en tant que tel, il est également présent comme partie du monde, une présence qui est une évocation de l'essentiel positionnement de l'être humain. Dans le temps en place, la place étant une qualité du monde, il y a une compétition dans laquelle ce qui émerge est le lieu de ce qui est politique. À un moment, Atê est à l'œuvre, et donc il y a l'œuvre du destin ; mais c'est également le domaine où le pardon trouve son expression - cette expression rendant compte de la présence du destin en même temps qu'elle situe la potentielle défaite de l'hégémonie du destin. Le pardon renvoie toujours la vengeance, en un renvoi qui doit être pensé comme une ouverture qui arrête l'œuvre d'Atê. Il introduit, en même temps qu'il la définit, la possibilité du «monde moral». La question est de savoir comment cette ouverture doit être comprise. Pour comprendre sa force, il est essentiel de tirer profit du terme auquel il a déjà été fait allusion, à savoir " césure ». Le terme joue un rôle fondamental dans les écrits de Benjamin depuis la première dissertation doctorale, Le concept de critique 
esthétique dans le romantisme allemand, jusqu'au Livre des passages ${ }^{15}$. La césure est une forme d'interruption radicale. Même s'il y a une interruption, elle a lieu dans un cadre. La césure est un éveil qui ne peut être assimilé ni à la destruction comme nihilisme, ni à la transformation comme forme de négation. Ce qui définit la césure dans ce contexte est ce qu'elle permet, ou en d'autres mots, ce qu'elle rend possible. Cependant, ce qu'elle rend possible, la forme de ceci, n'est pas soi-même donné à l'avance. La césure peut donc être pensée comme comprenant un moment intégré au fait de rendre le monde autre. Ce qui est rendu possible n'est pas une extension de la logique du monde préexistante. Le fait de rendre le monde autre dépend donc de ce qui sera désigné comme une "césure de la permission». Ce qui est central dans cette conception de l'interruption est double. Dans un premier temps, c'est l'ouverture à un espace d'activité. Dans un second temps, c'est le fait que ce qui est ouvert n'est pas déterminé par ce qui l'a rendu possible. C'est la force du terme "permettre». Il est ici entendu comme rendre possible et faire arriver. Le fait d'arriver en question ne peut pas être expliqué en termes de cause, ni de fondation déterminée (arché), ou jugé et évalué en termes de fin donnée (telos). Il y a là l'ouverture d'un processus et donc l'inauguration d'une « économie » définie par la continuité de l'activité.

Les lignes du paragraphe final du fragment se présentent de façon dramatique. Benjamin est bien sûr conscient de cette façon de faire, puisqu'il commence son dernier paragraphe en remarquant son propre usage de ce genre de langage, et continue en ajoutant qu'une telle manière de penser doit pouvoir être formulée de façon « claire et distincte dans une forme conceptuelle» (in Begriffen). La tempête "noie» le cri angoissé du «criminel » (Verbrecher), elle détruit les «traces » du «méfait » (Untat), et ceci a lieu même si la "Terre » (Erde) doit être ravagée par le processus. Cette tempête précède le Jugement dernier; celle-ci ne fait pas qu'avoir lieu avant le Jugement dernier. Le fait qu'elle ait lieu repousse le Jugement dernier là où celui-ci est compris comme la coïncidence du droit, de la finalité et de la vengeance. Elle éloigne Atê. C'est à ce point que Benjamin introduit une juxtaposition emphatique qui exige une reformulation attentive. Le pardon comme mouvement et comme processus est présenté dans des termes qui en font «la colère de Dieu», qui "rugit à travers l'histoire dans la tempête du pardon ». Ceci arrive afin d'emporter ce qui, sans cette tempête, aurait été "dévoré par les éclairs de la rage des Dieux» (den Blitzen des göttlichen Wetters). Les indications du processus de retardement sont composites. L'introduction du retardement doit être posée contre l'immédiateté. Celle-ci, comme indiqué plus haut, est l'œuvre du destin et donc des dieux. La vengeance n'est pas le résultat d'une décision potentiellement contestable. Elle est une finalité en acte qui a lieu sans médiation. Elle est le résultat de l'articulation du droit comme pure immédiateté, et par conséquent, l'interconnexion du destin, du droit et de la culpabilité.

20 Et alors se trouve la contre-mesure, le résultat de la contre-strophe, de la médiation. Intégrée au processus de la médiation, on trouve la proposition qui tient que la « fureur de Dieu » sauve. Elle sauve ce qui autrement aurait été exposé à la finalité du Jugement dernier, c'est-à-dire la finalité comme immédiate. Ce qui demande une plus grande clarification, c'est ce que ce cadre représente, à savoir « la signification du temps dans le monde moral ». Ce qui arrive dans ce «temps » est, comme les dernières lignes du fragment le disent clairement, ceci : d'abord le «temps » éteint les «traces » de tous les «méfaits». Ensuite, précisément du fait de sa durée temporelle, ce processus a lieu 
«par-delà tout souvenir ou tout pardon » (jenseits allen Gedenkens oder Vergessen). Puis, dans un troisième temps, ce positionnement est lui-même intégré au "processus du pardon» (Art zur Vergebung) - et il serait important de remarquer que le «pardon» n'est pas simplement défini par le moment où il arrive, car c'est, par définition, un " processus ». Finalement, ce qui n'est pas achevé est le processus de "réconciliation » (Versöhnung).

21 En dépit de la complexité de ces formulations, ce qui commence à émerger est une façon de comprendre la nature de la distinction entre le « droit » et le « monde moral». Deux points avec lesquels débuter: d'abord le fait que, plutôt que de les voir comme deux domaines distincts, le «droit» en premier lieu et le «monde moral» ensuite, il faut les considérer comme ayant lieu ensemble. Ils arrivent en rapport l'un avec l'autre. Et précisément parce qu'ils s'excluent mutuellement, il est nécessaire que là où il doit $\mathrm{y}$ avoir un monde moral, et donc du pardon, il y ait une suspension du droit au point que celui-ci soit identifié à l'immédiateté. Ainsi, dès le début, maintenant que sa base argumentative est établie, il est possible de réitérer l'assertion qui ouvre le texte : il n'y a pas de lien a priori entre la moralité et le droit. D'ailleurs, cette nécessité implique que toute tentative de promulguer ou de maintenir le pardon - et il ne peut y avoir que des tentatives pour la bonne et simple raison que le pardon arrive comme processus au sein d'un processus et donc comme médiation - est autant de fait pour susciter la suspension du droit. Le second point est que ce qui est impliqué ici est une contestation du temps. Ceci est un point qui est déjà présent dans la suggestion faite par Benjamin que le «temps» est le plus grand allié du pardon. Il y a un conflit en jeu entre la « tempête du pardon » et la « ruée du Jugement dernier ${ }^{16}$ ». Une fois que le « sursis » du Jugement dernier est pris comme central, le Jugement dernier lui-même envisagé comme le lieu d'un sursis potentiellement infini, arrivant dans le mouvement de la vengeance vers le pardon, ce qui s'ouvre alors est le vrai lieu du pardon. En d'autres termes, l'endroit est reconfiguré. Plutôt que la Terre comme endroit où le destin exerce son contrôle sur les décisions humaines et donc endroit qui serait également lieu des dieux, cet endroit devient le lieu de la décision et de la centralité des actions humaines. Cela signifie que ce qui arrive dans le mouvement du droit vers le pardon est l'ouverture de l'espace de la décision et du jugement. Le pardon dépend des relations entre espacement et immédiateté. Cet espacement, en tant que continuation de l'espace de la transformation, est la marque de ce qui est politique. C'est un point auquel il est fait allusion dans l'affirmation emphatique de Benjamin lui-même qui tient que dans la tempête du pardon les traces des méfaits seront effacées, "quand bien même il lui faudrait pour cela dévaster (verwüsten) la terre ».

Étant donnée cette description, ce qu'il faut chercher c'est comment la référence à la "Terre» (Erde) doit être comprise. La dévastation de la Terre est ce qui fait "disparaitre les traces" des "méfaits". Cependant, il ne s'agit pas d'une simple destruction, cette dernière serait la forme de destruction exigée par la culpabilité et mise en œuvre au sein de différentes formes de finalité. Il s'agit plutôt ici de la destruction du pardon. C'est l'autre possibilité de la destruction. La «Terre», en tant que terme du texte de Benjamin, ne peut être comprise de façon adéquate que si elle est envisagée comme désignant ce qui a déjà été décrit, comme le fait pour les êtres humains d'être placés. La Terre devient, en conséquence, ce qui continue dans la continuité du pardon. Le pardon, précisément parce qu'il ne relève pas de la complexe interaction entre destin, droit et vengeance, cadre qui se définit en termes d'immédiateté, car il demande bien la suspension de l'immédiateté, est toujours en 
place. Le pardon est une potentialité déjà présente. La "Terre » est ainsi maintenue en place par le fait d'être devenue le lieu de la moralité; la moralité émerge avec la continuité de ce qui est dans la médiation. La moralité, comme il a déjà été indiqué, dépend de la suspension du droit et, par conséquent, est positionnée par-delà le nihilisme de la destruction. À l'œuvre au sein de l'ouverture de la moralité, à l'œuvre en son sein précisément parce qu'elle devient la condition préalable de la moralité, se trouve une suspension qui devra toujours être reconfigurée en termes de césure de ce qui rend possible, précisément parce que ce qui est rendu possible est l'identification de la Terre comme l'espace de la médiation. Le monde ne pourra plus déchoir. Le monde moral est le monde devenu sacré.

Si le « pardon " nécessite des moments d'accomplissement - il doit y avoir pardon, des décisions sont prises -, il y a un élément supplémentaire. Car, comme le suggère Benjamin, ce qui n'est pas mené à son terme est le processus de "réconciliation ». Cette dernière est la vie continuée après avoir pardonné. Pardonner ne se fait pas en une fois. Alors que ce que Benjamin pourrait avoir voulu dire par « réconciliation» peut être débattu, il est cependant toujours possible de lier la nature incomplète de la réconciliation, telle qu'exigée par le pardon comme processus, au renvoi permanent du Jugement dernier, et donc de la situer comme l'endroit de l'activité mortelle (humaine) du pardon ${ }^{17}$ - dans ce cadre, le Jugement dernier est compris au sens restreint de ce qui est exigé par le rapport entre droit et vengeance. Le pardon est une instance de la présence opérative de la médiation et donc de «l'économie du monde moral ». En tant que telle, la nature incomplète de la réconciliation nomme le lieu de la décision et réitère le rapport déjà identifié entre espacement et médiation. Cette dernière est, bien sûr, l'endroit de ce qui est politique. Ce que cela signifie, c'est que la connexion entre ce qui est politique et ce qui est moral (compris comme impliquant le nécessairement incomplet, où l'incomplétude et la médiation ont une co-présence nécessaire, en tant que cela nomme le processus et donc une économie morale) est rendue possible par la suspension du rapport entre droit et destin. Ceci affine un peu plus la formulation initiale de la jonction où la structure de la critique peut être située.

Benjamin, dans un texte écrit quelques années avant ce fragment et qui a été préservé par Gershom Scholem dans son Journal, à savoir Notizen zu einer Arbeit über die Kategorie der Gerechtigkeit, a noté ce qui suit en rapport avec la justice :

Gerechtigkeit scheint sich nicht auf den guten Willen des Subjekts zu beziehen, sondern macht einen Zustand der Welt aus. Gerechtigkeit bezeichnet die ethische Kategorie des Existenten, Tugend die ethische Kategorie des Geforderten. Tugend kann gefordert werden, Gerechtigkeit letzten Endes nur sein, als Zustand der Welt oder als Zustand Gottes.

La justice n'apparait pas être en relation avec la bonne volonté d'un sujet, elle est plutôt une partie de l'état du monde. La justice désigne la catégorie éthique de l'existant, et la vertu, la catégorie éthique de ce qui est exigé. La vertu peut être exigée, la justice peut au final exister seulement comme un état du monde, ou bien un état de Dieu ${ }^{18}$.

La double insistance sur « le monde » et sur « l'existence » est ici centrale. Un peu plus tôt dans le texte, tel que le transcrit Scholem, le rapport entre "la justice » et «le monde » est formulé dans les termes suivants : "Gerechtigkeit ist das Streben, die Welt zum höchsten Gut zu machen" (La justice est l'effort pour élever le monde au bien suprême ${ }^{19}$ ). Le monde n'est pas donné. Tant le monde qu'un souci de l'existence ne peuvent entrer 
en considération qu'avec la suspension de la présence effective de la culpabilité, le domaine des dieux, à savoir le monde en tant qu'il est déjà déterminé à l'avance, et ce qui finalement émergera des écrits de Benjamin comme le monde de l'historicisme, un monde conservatoire du même. Ainsi, dans ce contexte, ceci rend compte de l'importance de la référence à Atê. Afin de rassembler certains des thèmes déjà évoqués, un retour doit être fait à la figure d'Atê. Alors que de nombreux contextes sont possibles, la présence d'Atê chez Homère souligne ce qui est en jeu.

Dans l'Iliade, pour défendre ses actes, après s'être vu reprocher par les Achéens d'avoir volé Achille, Agamemnon répond qu'il n'est pas en faute. Il n'était pas la cause de ce qui lui était arrivé. Sa vue avait été aveuglée. De ce fait, il ne pouvait pas discerner, au moment de commettre de tels actes, le bien du mal. Les fins ne sont pas l'œuvre des humains (mortels). Au contraire - comme l'Iliade le montre clairement - «ce sont les Dieux qui mènent les choses selon leurs fins ${ }^{20} »$. Agamemnon continue sa défense en situant la cause réelle chez Atê : «Fille aînée de Zeus, Atê qui aveugle tous - Maudite ${ }^{21}$ ». La figure d'Atê montre avec emphase que le monde des mortels, le monde comme endroit de la décision, ne peut émerger qu'à la condition que les dieux ne définissent plus le monde comme lieu de la décision. Dans un tel mouvement, la décision en tant que réponse immédiate cède sa place à la décision en tant que ce qui émerge de la médiation intrinsèque du jugement. En d'autres termes, c'est la suspension de cette conception du monde, le monde de l'immédiateté, qui seule rend possible l'émergence d'un monde défini comme lieu de ce qui est en place, potentialité qui est inhérente au fait de l'existence dans le monde. Ce fait a déjà été remarqué dans l'assertion de Benjamin qui tient que le «jugement existe en tant qu'état du monde». Ceci constitue de plus le fait de rendre le monde autre selon Marx. Dans le même passage de l'Iliade, la relation causale qui a pour résultat des événements dans le monde est toujours dégagée du domaine humain. Le rôle déterminant dans ce que l'on prend pour des actions humaines, qui sont donc envisagées comme le résultat de décisions, là où la décision elle-même structure le domaine dans lequel la responsabilité et le jugement seraient à l'œuvre, ce rôle est donné au destin (Moira). Le destin est présent à la fois sous une forme personnifiée, et comme temps. Au sujet de ce dernier, c'est également le temps sur lequel les humains ne peuvent exercer leur contrôle. Ils sont les sujets du destin. Ainsi, quoique présents, ils ne sont pas seulement prédestinés en tant que sujets, mais aussi positionnés au sein d'une structure de culpabilité originelle.

Ce qui est introduit ici, au sein du monde ouvert par la figure d'Atê telle qu'elle arrive dans l'Iliade, est une distinction temporelle entre, d'une part, l'éternité du destin, une éternité dans laquelle les détails se produisent, dans laquelle l'histoire en tant qu'histoire des événements a lieu, et d'un autre côté, un domaine d'activité et donc d'histoire (une autre conception de l'histoire) qui ne peut arriver qu'avec la suspension du premier. Cependant, il est à noter que cette suspension ne réduit pas les actions humaines à ce qui est pragmatique - ce qui serait traité dans le contexte d'un souci du jugement des actions humaines sous le titre de «droit positif ${ }^{22}$ ». Cela nécessite plutôt de repenser ce qui fonde le jugement, la responsabilité et donc la décision. Et, ce faisant, vient inévitablement ce qui a déjà été décrit comme le fait pour l'être humain d'être placé; c'est-à-dire l'être humain comme être placé. Il faut reconnaître, par ailleurs, que le domaine du rapport entre destin et particularité devra toujours être suspendu. Le fait de suspendre devient une activité précisément parce qu'il implique la continuité de l'action - la continuité de la discontinuité - et par là-même ne peut être 
compris dans les termes d'une logique de négation. De la même façon, quoique de l'autre perspective, il se tient face à la temporalité de l'éternel retour. Il y a, par conséquent, la possibilité continuelle d'une forme de retour. Un tel retour n'arriverait pas au nom des dieux; mais plutôt ce qui continue de durer est l'interaction entre le destin en tant que conception du temps historique et politique et la conception du droit qui en découle d'un côté, et de l'autre la création continue de ce qui arrive au sein de l'espace ouvert par la césure de ce qui rend possible. Un exemple de cette dernière est le "processus du pardon " déjà évoqué. Ce qui est ouvert, par le déplacement depuis l'immédiat vers l'espace de la médiation n'est pas simplement le « monde moral », c'est aussi la possibilité de la critique. Ce que cela signifie, c'est que la critique devient possible dans la mesure où le fait de rendre le monde autre a lieu. Cependant, précisément parce que le fait de rendre le monde autre est la recréation du monde comme «monde moral », il n'est pas établi comme un événement unique. Ce qui passe au premier plan est un monde dans lequel la justice, plutôt que d'être liée soit à un serment, soit à ce qui est transcendant, est "un état du monde ». Le fait de rendre le monde autre doit être compris comme une continuité de la pratique. Une continuité dans laquelle le lieu de la critique, et ceci sera également le cas avec la justice, est le monde dans la continuité du fait d'être rendu autre. Le fait de rendre autre devient l'assertion qui tient que la justice est affirmée comme qualité du monde. Pourquoi ? Parce qu'exister et exister de façon juste ont la possibilité d'être synonymes.

Renforcer l'affirmation qui tient que cette potentialité est une qualité déjà présente au sein du monde, et qui peut être recouvrée depuis le monde de par la suspension du droit - ce qui est après tout la jonction dans laquelle la structure de la critique doit être trouvée -, telle est la position tenue par Benjamin qui peut être remarquée dans des suggestions faites presque en passant dans la Critique de la violence ${ }^{23}$. L'affirmation de Benjamin est que ce qu'il appelle "pouvoir éducateur» (erzieherische Gewalt) a le "pouvoir d'annihiler par la destruction de toute création de droit ${ }^{24}$ ». La force de ce passage est double. Là où elle peut être située tout d'abord, elle est l'identification d'une forme de "pouvoir» (Gewalt) qui a le potentiel pour provoquer une suspension de l'œuvre de l'immédiateté, là où le pouvoir est situé, dans un cadre qui porte avec lui la possibilité, et donc la potentialité, de ne pas avoir été de tout temps et inévitablement - et donc fatalement - structuré par la logique réelle du monde. Ensuite, elle est le lien que cette conception du "pouvoir » a avec les formes d'accord qui ont leur base dans la "sympathie » et la "confiance». En d'autres termes, il y a dans ce qui pourrait être décrit comme l'étoffe de l'existence, un potentiel dans cette étoffe, la possibilité que, étant donnée la suspension du droit (où ce dernier est compris en termes d'immédiateté), il y ait d'autres formes de relationnalité. Elles doivent être rendues possibles.

La critique est le nom de la jonction au sein de laquelle la césure, du fait de rendre possible, vient à être liée autant à une rencontre avec ce qui est philosophique et avec l'œuvre d'art, qu'aux relations sociales. Une fois que le monde n'est plus, soit réductible à ce qui est uniquement conceptuel, soit pris comme explicable en termes d'hypothèse de sa qualité de il y a-qualité qui se déploie autant en rapport avec le fait de poser l'immutabilité de la structure qu'avec sa réduction à ce qui est purement empirique -, alors la critique est inextricablement liée à l'affirmation de la potentialité du monde à être autre. 


\section{BIBLIOGRAPHIE}

Benjamin Andrew, The Plural Event: Descartes, Hegel, Heidegger, London, Routledge, 1993.

Benjamin Andrew, "Time and Task: Benjamin and Heidegger Showing the Present", in Present Hope: Philosophy, Architecture, Judaism, A. Benjamin, London, Routledge, 1997, p. 26-55.

Benjamin Andrew, Architectural Philosophy, London, Athlone Press, 2000.

Benjamin Andrew, "Boredom and Distraction. The Moods of Modernity", in Walter Benjamin and History, A. Benjamin (ed.), London, Continuum, 2005, p. 156-170.

Benjamin Andrew, "Benjamin's Modernity”, in Style and Time: Essays on the Politics of Appearance, A. Benjamin, Evanston (Ill.), Northwestern University Press, 2006, p. 5-24.

Benjamin Andrew, “Messianic Nature: Notes on Walter Benjamin's Theological-Political Fragment", in Limbus: Australisches Jahrbuch für germanistische Literatur und Kulturwissenschaft, Australian Yearbook of German Literary and Cultural Studies, 2010.

Benjamin Andrew, Place, Commonality and Judgment: Continental Philosophy and the Ancient Greeks, London, Continuum, 2010.

Benjamin Walter, Gesammelte Schriften, Band VI, Rolf Tiedemann, Hermann Schweppenhaüser (ed.), Francfort am Main, Suhrkamp, 1985.

Benjamin Walter, « Destin et caractère » [1919], in CEuvres I, Paris, Gallimard (Folio Essais), 2000, p. 198-209.

Benjamin Walter, « Fragment théologico-politique », in Euvres I, Paris, Gallimard (Folio Essais), 2000, p. 263-265.

Benjamin Walter, « La signification du temps dans le monde moral » [1921], in Fragments, Paris, PUF/Librairie du Collège international de philosophie, 2001.

Benjamin Walter, Selected Writings, vol. I, Howard Eiland, Michael W. Jennings (ed.), Cambridge (Mass.), Harvard University Press, 2002.

Birnbaum Antonia, Bonheur justice, Walter Benjamin, Paris, Payot, 2008.

Cohen Hermann, Ethik des Reinen Willens, Berlin, Bruno Cassirer, 1904.

Cottingham John, Cartesian Reflections: Essays on Descartes's Philosophy, Oxford, Oxford University Press, 2008.

Derrida Jacques, Force de loi, Paris, Galilée, 1994.

Descartes René, Cuvres, Charles Adam, Paul Tannery (éd.), Paris, Vrin, 1996.

Descartes René, Meditations on First Philosophy, Michael Moriarty (trad.), Oxford, Oxford University Press, 2008.

Deuber-Mankowsky Astrid, "The Ties between Walter Benjamin and Hermann Cohen: A Generally Neglected Chapter in the History of the Impact of Cohen's Philosophy", Joel Golb (trad.), Journal of Jewish Thought and Philosophy, vol. XIII, n 1-3, 2004, p. 127-145.

Fenves Peter, The Messianic Reduction: Walter Benjamin and the Shape of Time, Stanford (Calif.), Stanford University Press, 2011. 
Gillespie Michael A., The Theological Origins of Modernity, Chicago (Ill.), University of Chicago Press, 2008.

Hegel Georg Wilhelm Friedrich, "The Spirit of Christianity and its Fate”, in Early Theological Writings, G. W. F. Hegel, T. M. Knox (trad.), Philadelphie (Penn.), University of Philadelphia Press, 1971, p. 182-301.

Hegel Georg Wilhelm Friedrich, "Der Geist des Christentums", in Frühe Schriften, G. W. F. Hegel, vol. I, Francfort am Main, Suhrkamp, 1986, p. 317-418.

Hegel Georg Wilhelm Friedrich, L'esprit du christianisme et son destin, Paris, Vrin, 2000.

Heidegger Martin, Grundfragen der Philosophie, Francfort am Main, Vittorio Klostermann (Gesamtausgabe ; $\left.\mathrm{n}^{\circ} 45\right), 1992$.

Homère, Iliad, A.T. Murray (trad.), William F. Wyatt (ed.), Cambridge (Mass.), Harvard University Press, 1999.

Kambouchner Denis, Les Méditations métaphysiques de Descartes, Paris, PUF, 2005.

Marx Karl, Engels Friedrich, « Thèses sur Feuerbach », in L'idéologie allemande, Gilbert Badia (éd.), Paris, Éditions sociales, 1976.

Marx Karl, “Theses on Feuerbach", in Selected Writings, Karl Marx, Lawrence H. Simmon (ed.), Indianapolis, Hackett, 1987. Thesen über Feuerbach, pour un fac-similé du manuscrit original, voir : http://www.mlwerke.de/me/me03/me03_533.htm

Nonnos, Dionysiaca, Books 1-15, W. H. D. Rouse (trad.), Cambridge (Mass.), Harvard University Press, 1940.

Noor Ashraf, "Walter Benjamin. Time and Justice”, in Naharaim: Zeitschrift für deutsch-jüdische Literatur und Kulturgeschichte, vol. I, $\mathrm{n}^{\circ} 1,2007$, p. 38-74.

Osborne Peter, The Politics of Time: Modernity and Avant-Garde, London, Verso, 1995.

Scholem Gershom, Tagebücher 1913-1917, Karlfried Grunder, Herbert Kopp-Oberstebrink, Friedrich Niewöhner (ed.), Francfort am Main, Jüdischer Verlag, 1995.

Scholem Gershom, Tagebücher 1917-1923, Karlfried Grunder, Herbert Kopp-Oberstebrink, Friedrich Niewöhner (ed.), Francfort am Main, Jüdischer Verlag, 2000.

Tomba Massimiliano, “Another Kind of Gewalt: Beyond Law. Re-Reading Walter Benjamin”, Historical Materialism, vol. XVII, n 1, 2009, p. 126-144. http://dx.doi.org/

$10.1163 / 156920609 \times 399236$

Weber Samuel, Benjamin's - Abilities, Cambridge (Mass.), Harvard University Press, 2008.

\section{NOTES}

2. Il est à remarquer que Descartes, lorsqu'il écrit en latin, emploie le terme eversio qui se traduit plus directement par « renversement » (overturning), alors que la traduction d'époque du latin vers le français - assurée par le duc de Luynes en 1647 et approuvée par Descartes - est «à détruire ", plus appuyée. Voir René Descartes, CEuvres, Charles Adam, Paul Tannery (éd.), Paris, Vrin, 1996. Pour le latin voir t. VII, p. 18 et pour le français, t. IX, p. 13. Pour un examen de eversio/destruction au sein même des termes du projet de Descartes, voir Denis Kambouchner, Les Méditations métaphysiques de Descartes, Paris, PUF, 2005, p. 191-201. Il est intéressant de noter que la traduction récente la plus importante des Méditations en anglais, à savoir René Descartes, 
Meditations on First Philosophy, Michael Moriarty (trad.), Oxford, Oxford University Press, 2008, choisit « à détruire » (destroying) plutôt que " renversement », voir p. 13. Pour une interprétation différente et plus poussée de cet acte de «destruction», voir Michael Allen Gillespie, The theological Origins of Modernity, Chicago (Ill.), University of Chicago Press, 2008, p. 170-207. Il y a, bien sûr, des interprétations d'ensemble du projet philosophique de Descartes qui, tout en notant le rôle de la "destruction", ne lui attribuent de rôle central dans aucune interprétation ultérieure. Comme exemple d'une telle approche, voir John Cottingham, Cartesian Reflections: Essays on Descartes's Philosophy, Oxford, Oxford University Press, 2008, p. 53-57.

3. J'ai présenté une analyse montrant les points d'intersection et de différenciation entre les conceptions de la destruction chez Benjamin et Heidegger dans "Time and Task: Benjamin and Heidegger Showing the Present", in Present Hope: Philosophy, Architecture, Judaism, London, Routledge, 1997, p. 26-55. Il est à noter cependant que la thématique de la "destruction » chez Heidegger se trouve remplacée par une pensée continue de la fin (das Ende) et de ses rapports aux « commencements ». Voir à ce sujet les $§ 31$ et suivants du livre de Martin Heidegger, Grundfragen der Philosophie, Francfort am Main, Vittorio Klostermann, (Gesamtausgabe, n ${ }^{\circ}$ 4), 1992.

4. Cette expression trouve son origine dans l'œuvre de Peter Osborne, The Politics of Time: Modernity and Avant-Garde, London, Verso, 1995. Si l'acuité de la vision d'Osborne sur les rapports entre la politique et les diverses conceptions du temps historique ne peut être prise en défaut, le point de discorde, autour de Benjamin, concerne l'argument d'Osborne qui tient que le mouvement du temps ne peut être compris comme un appareil qui met en scène la fin de la narration depuis l'intérieur des confins de la narration elle-même. À ce sujet, voir le livre d'Osborne, p.157. En somme, l'interruption requiert une conception de l'internalité et de l'externalité plus complexe, qui est saisie par la restriction de l'argument à la structure de la narration.

5. In Walter Benjamin Selected Writings, vol. I, Howard Eiland, Michael W. Jennings (ed.), Cambridge (Mass.), Harvard University Press, 2002, p. 286-288. En allemand voir: Walter Benjamin, Gesammelte Schriften, Band VI, Rolf Tiedemann, Herman Schweppenhaüser (ed.), Francfort am Main, Suhrkamp, 1985, p. 97-98. Toutes les références suivantes au texte de Benjamin seront basées sur l'édition en anglais, suivie de celle en allemand, et indiquées dans le corps du texte. Cependant, en cohérence avec l'insistance sur la centralité du monde, le titre du texte Die Bedeutung der Zeit in der moralische Welt est compris en termes de souci du «monde moral » et non d'« univers moral » comme l'indique la traduction des Selected Writings.

6. "Towards a Critique of Violence" de Benjamin a suscité une énorme littérature secondaire. Le projet ici n'est pas de discuter de ce texte. Cependant, pour une recherche qui souligne les éléments clés dans ce texte et son rapport à la question de la "violence » (Gewalt), voir Massimiliano Tomba, “Another Kind of Gewalt: Beyond Law Re-Reading Walter Benjamin”, Historical Materialism, vol. XVII, $\mathrm{n}^{\circ} 1,2009, \mathrm{p} .126-144$.

7. J'ai tout d'abord argumenté en faveur de la centralité du concept de potentialité pour comprendre l'œuvre de Walter Benjamin dans l'article "Boredom and Distraction: The Moods of Modernity", in Walter Benjamin and History, Andrew Benjamin (ed.), London, Continuum, 2005. J'ai développé cette position dans plusieurs écrits récents sur Benjamin. La façon dont la potentialité est utilisée dans cet article, et ailleurs dans mon travail sur Benjamin, concerne la présence d'une pensée non nommée, mais qui est pour autant un concept indispensable, à l'œuvre dans les écrits de Benjamin. Il est à noter qu'une position similaire, quoique argumentée de façon fort différente, a également été avancée par Samuel Weber dans son livre Benjamin's-Abilities, Cambridge (Mass.), Harvard University Press, 2008.

8. (C'est Marx qui souligne). Pour le texte allemand original, voir Thesen über Feuerbach en facsimilé du manuscrit original : http://www.mlwerke.de/me/me03/me03_533.htm

9. Karl Marx, Friedrich Engels, "Thèses sur Feuerbach », in L'idéologie allemande, Gilbert Badia (éd.), Paris, Éditions sociales, 1976. 
10. La catastrophe est une contre-strophe et sera envisagée comme la structure d'une césure. Ce point sera développé en détail dans l'argumentation à venir.

11. Walter Benjamin, « La signification du temps dans le monde moral », p. 107-108.

12. Walter Benjamin, «Destin et caractère » [1919], in CEuvres I, Paris, Gallimard (Folio Essais), 2000 , p. 203.

13. Ibid., p. 202.

14. Walter Benjamin, Fragments, p. 108.

15. J'ai traité des emplois variés du terme «césure » par Walter Benjamin dans "Benjamin's Modernity", in Style and Time Essays on the Politics of Appearance, Evanston (Ill.), Northwestern University Press, 2006, p. 5-24.

16. Ce jeu de forces quasi chiasmatique réitère la position annoncée par Walter Benjamin dans le "Fragment théologico-politique », in Euvres I, Paris, Gallimard (Folio Essais), 2000, p. 264.

L'ordre du profane doit s'édifier sur l'idée de bonheur. La relation de cet ordre avec l'élément messianique est l'un des enseignements essentiels de la philosophie de l'histoire. Cette relation conditionne en effet une conception mystique de l'histoire, dont le problème se peut exposer en une image. Si l'on représente par une flèche le but vers lequel s'exerce la dunamis du profane, par une autre flèche la direction de l'intensité messianique, assurément la quête du bonheur de la libre humanité tend à s'écarter de cette orientation messianique. Mais de même qu'une force peut, par sa trajectoire, favoriser l'action d'une autre force sur une trajectoire opposée, ainsi l'ordre du profane peut favoriser l'avènement du royaume messianique.

J'ai traité de ce jeu de forces dans l'article "Messianic Nature: Notes on Walter Benjamin's 'Theological-Political Fragment' ", in Limbus: australisches Jahrbuch für germanistische Literatur und Kulturwissenschaft. Australian Yearbook of German Literary and Cultural Studies, 2010.

17. Le langage du «pardon» est bien sûr celui du christianisme. Chez Benjamin cependant, on peut arguer du fait que le renvoi de la réconciliation est lié au refus du pardon immédiat. Le pardon immédiat est bien sûr la position que Hegel attribue au Christ. Son commentaire sur Luc, VII, 48 est décisif à cet égard. Hegel écrit :

«Cette sentence n'est pas l'annulation objective du châtiment, la destruction d'un destin qui subsistait encore [kein Zerstören des nicht bestehenden Schicksals], mais seulement le fait de la confiance de Jésus qui, dans la foi de ceux qui le comprennent, s'est reconnu lui-même, reconnaît des cœurs semblables au sien, lit dans leur foi leur élévation au-dessus de la loi et du destin [Gesetz und Schicksal], et leur annonce le pardon de leurs péchés. " L'esprit du christianisme et son destin, Paris, Vrin, 2000, p. 60-61.

Ce qui est à l'œuvre ici est complexe. " Le droit [la loi] et le destin » ne sont dépassés qu'à travers un acte de contre-immédiateté. Plus encore la conception du droit qui est en jeu dans la formulation de Hegel est la production du droit comme immédiat. Ceci peut être décrit comme le fait de rendre le droit littéral. J'ai essayé de montrer que ceci marque une séparation entre le droit et la justice qui a lieu tout d'abord chez Paul et qui, ce faisant, défait une identification du droit et de la justice qui a lieu tout au long de la pensée grecque. La suspension du droit par Benjamin est la suspension du droit comme immédiat afin de créer un repositionnement de celuici comme intrinsèquement médiat. Si c'était possible, cela permettrait une réarticulation du rapport entre droit et justice. Voir, de l'auteur du présent article, Place, Commonality and Judgment: Continental Philosophy and the Ancient Greeks, London, Continuum, 2010.

18. Gershom Scholem, Tagebücher 1913-1917, Karlfried Grunder, Herbert Kopp-Oberstebrink, Friedrich Niewöhner (ed.), Francfort am Main, Jüdischer Verlag, 1995, p. 401.

19. Ibid., p. 401. Il est également à noter que le terme « effort » joue un rôle fondamental dans le Fragment théologico-politique.

20. In Homère, Iliade, A.T. Murray (trad.), W. F. Wyatt (ed.), Cambridge (Mass.), Harvard University Press, 1999, XIX, 90.

21. Ibid., XIX, 91-92. 
22. Ici, l'intérêt propre de Walter Benjamin pour la tradition, tant du droit naturel que du droit positif, comme le montrent les premières pages de la Critique de la violence, serait d'une importance centrale. Voir Walter Benjamin, Cuvres I, p. 210 et suiv.

23. En effet, si des passages de cette nature devaient être mis en avant, ils permettraient alors d'endiguer cette identification au sein de l'œuvre de Benjamin d'une philosophie de la violence réelle. Une telle interprétation se trouve, par exemple, dans le livre de Jacques Derrida, Force de loi, Paris, Galilée, 1994.

24. Walter Benjamin, CEuvres I, p. 214.

1. Walter Benjamin, «La signification du temps dans le monde moral » [1921], in Fragments, Paris, PUF/Librairie du Collège international de philosophie, 2001.

\section{RÉSUMÉS}

La critique, en tant que concept philosophique, doit être refondue lorsqu'elle est reliée à la possibilité d'une ouverture productive. Dans un tel contexte, la critique a une grande affinité avec la destruction et avec des formes d'inauguration. En travaillant au fil des écrits de Marx et de Walter Benjamin, et plus spécifiquement autour de «La signification du temps dans le monde moral"1 de Benjamin, la destruction et l'inauguration sont repositionnées en termes d'altérité et de césure de la permission.

\section{INDEX}

Mots-clés : césure, critique, destruction

Personnes citées : Marx (Karl), Benjamin (Walter)

\section{AUTEURS}

\section{ANDREW BENJAMIN}

Andrew Benjamin est professeur de philosophie à l'Université Monash, à Melbourne. Tourné vers la philosophie européenne, il a notamment travaillé sur Heidegger, Adorno, W. Benjamin, Lyotard. Il a développé également toute une recherche en architecture, en liaison avec son propre enseignement, dans plusieurs écoles d'architecture (Royaume-Uni, États-Unis, Australie). 